\title{
СИСТЕМА ТРИАНГУЛЯЦИОННЫХ ЛАЗЕРНЫХ ДАЛЬНОМЕРОВ ДЛЯ РОБОТИЗИРОВАННОЙ ОБРАБОТКИ С ИСПОЛЬЗОВАНИЕМ МЕТОДОВ ТЕХНИЧЕСКОГО ЗРЕНИЯ 1
}

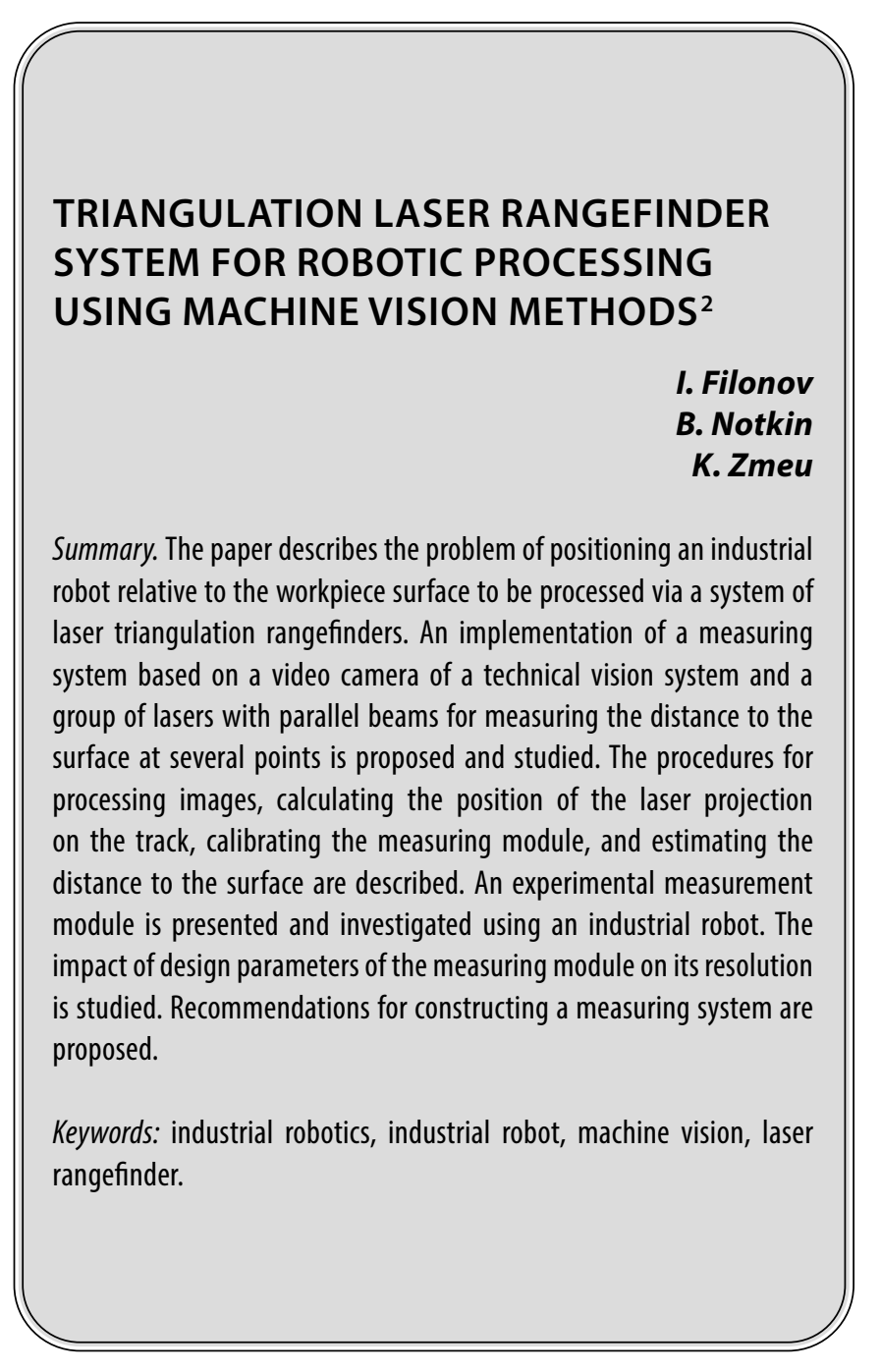

\section{Введение}

B области промышленной робототехники широко используются системы технического зрения для решения различных задач визуального контроля и диагностики $[1,2,3,4]$. Часто эти задачи совмещены с необходимостью позиционирования робота относительно наблюдаемого объекта и его окружения
Филонов Иван Владимирович

Аспирант, ФГАОУВО «Дальневосточный федеральный университет», (Владивосток)

Filonov.iv@dvfu.ru

Ноткин Борис Сергеевич

K.т.н, н.с., ФГБУН «Институт автоматики и прочессов управления Дальневосточного отделения

Российской академии наук», (Владивосток) notkin@iacp.dvo.ru

Змеу Константин Витальевич K.т.н, дочент, ФГАОУ ВО «Дальневосточный федеральный университет», (Владивосток) zmeu.kv@dvfu.ru

Аннотация. В работе рассматривается задача позиционирования промышленного робота относительно обрабатываемой поверхности детали с помощью системы лазерных триангуляционных дальномеров. Предложена и исследована реализация измерительной системы, построенная на базе видеокамеры системы технического зрения и группы лазеров с параллельными лучами для измерения расстояния до поверхности в нескольких точках. Описаны процедуры обработки изображений, расчета положения проекции лазера на треке, калибровки измерительного модуля, оценки расстояния до поверхности. Представлен экспериментальный модуль измерения, проведено его исследование с помощью промышленного робота. Исследовано влияние конструктивных параметров измерительного модуля на его разрешающую способность. Предложены рекомендации по построению измерительной системы.

Ключевые слова: промышленная робототехника, промышленный робот, техническое зрение, триангуляционный лазерный дальномер.

[5,6,7,8]. Для этого могут использовать как средства обработки и анализа изображений [9], регистрируемых видеокамерой системы технического зрения, так и дополнительные измерительные устройства. Наиболее часто в качестве таких дополнительных устройств используются лазерные триангуляционные дальномеры (ЛТД). Можно выделить класс задач, где измерение расстояния требуется выполнять одновре- 


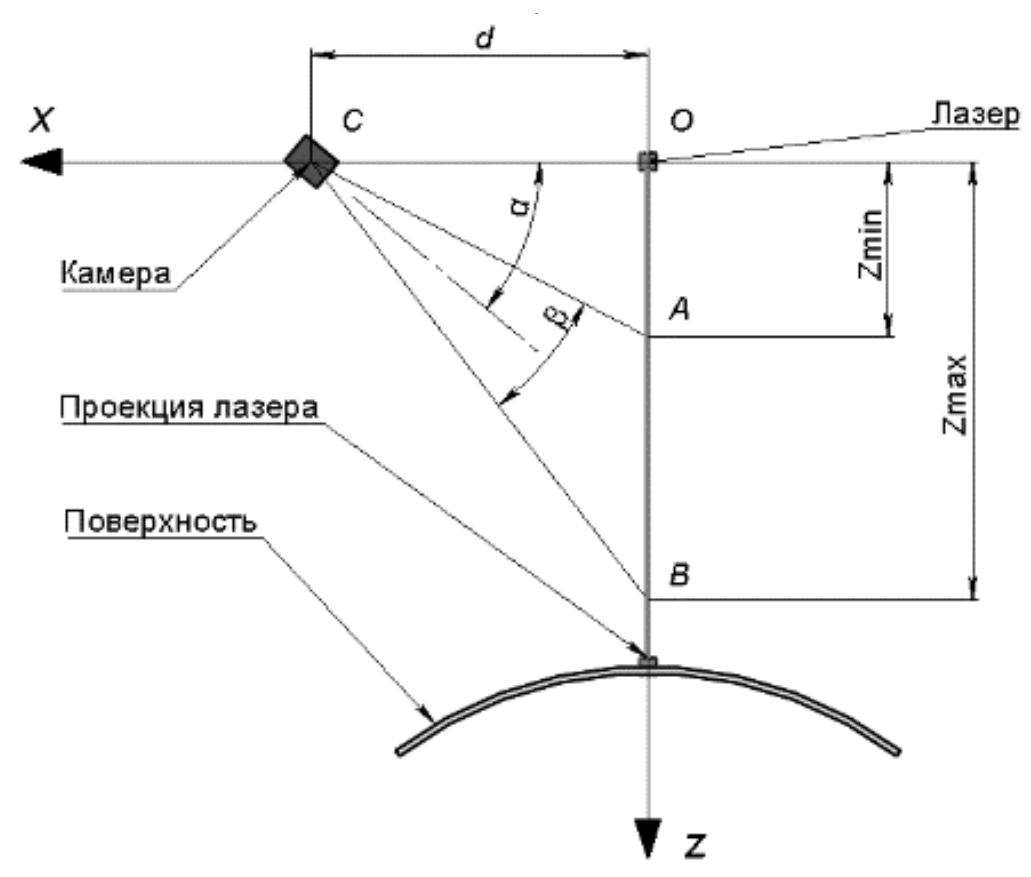

Рис. 1. Схема модуля измерения

менно в нескольких точках. В области промышленной робототехники примерами таких задач являются задачи позиционирования инструмента относительно обрабатываемой поверхности, например, при сверлении или клепке $[15,16,17,13]$, где важно контролировать не только положение инструмента, но и его ориентацию, часто по нормали к поверхности. В таких решения может использоваться от трех модулей лтД [21] и более [20]. Возможны различные конфигурации размещения ЛТД, отличающиеся как рисунком (например, ромб [11] или круг [19]), так и взаимной ориентаций лучей (параллельные друг другу [12] или наклонные [10,14,18]). В настоящей работе предлагается и исследуется реализация измерительной системы, построенная на базе камеры системы технического зрения, которая

дополнена группой лазеров с параллельной ориентацией лучей. Эксперименты выполнены с использованием промышленный робота KUKA KR10 R900 и веб-камеры.

Работа организована следующим образом. В разделе 1 рассматривается измерительная система на базе одного лазера и видеокамеры. В разделе 2 на примере этой системы устанавливается зависимость координаты проекции лазера в кадре камеры от изменения расстояния до поверхности. В разделе 3 представляется реализация многолучевой измерительной системы на базе группы параллельных лазеров. Раздел 4 содержит результаты экспериментального исследования лабораторного образца измерительного модуля, размещенного на фланце промышленного робота.

\section{1. Конструктивные параметры

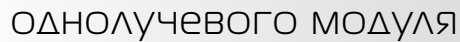 измерения расстояния}

Для измерения расстояния до поверхности используется метод лазерной триангуляции, в соответствии с которым, можно измерить расстояние от модуля измерения до проекции лазерного луча на поверхности при известных координатах расположения элементов оптической системы - лазера и камеры [13]. Конструкция модуля измерения представлена на рисунке 1

Рабочий диапазон модуля измерения ограничен зоной видимости проекции луча в кадре камеры. На рисунке 1 он обозначен интервалом $z_{\min }$ и $z_{\max }$. основные конструктивные параметры модуля измерения зависит от угла обзора камеры $\beta$, её положения $d$ и наклона $\alpha$.

Выражая косинус угла обзора камеры $\beta$ через скалярное произведение векторов $\overline{C A}=\left(d, z_{\min }\right)$ и $\overline{C B}=\left(d, z_{\max }\right)$, запишем уравнение (1), связывающее основные конструктивные параметры модуля измерения

$$
\begin{aligned}
& d^{2}+z_{\min } \cdot z_{\max }= \\
& =\sqrt{d^{2}+z_{\min }^{2}} \cdot \sqrt{d^{2}+z_{\max }^{2}} \cdot \cos \beta .
\end{aligned}
$$

При изменении расстояния от модуля измерения до поверхности по координате $z$ происходит переме- 
щении проекции лазера в кадре камеры. Множество таких проекций на интервале рабочего диапазона от $z_{\text {min }}$ до $z_{\text {max }}$ образуют в кадре камеры трек.

\section{2. Зависимость проекции лазера в калре камеры от расстояния $\triangle$ о поверхности}

В соответствии с моделью pinhole camera [14], координаты проекции лазера $u, v$ в кадре камеры будут иметь следующий вид:

$$
\begin{aligned}
& u=f \cdot \frac{x_{\mathrm{c}}}{z_{\mathrm{c}}}+c_{x} ; \\
& v=f \cdot \frac{y_{\mathrm{c}}}{z_{\mathrm{c}}}+c_{y},
\end{aligned}
$$

где $x_{c}, y_{c}, z_{c}$ - координаты проекции лазера на поверхности в системе координат камеры, $c_{x}, c_{y}-$ координаты главной точки оптической оси кадра камеры.

Вектор положения проекции лазера $P$ в системе координат измерительного модуля:

$$
P=\left|\begin{array}{l}
x \\
y \\
z
\end{array}\right|=\left|\begin{array}{l}
0 \\
0 \\
z
\end{array}\right|,
$$

где согласно схеме на рисунке 1, координаты $x$ и $y$ проекции луча лазера равны нулю, так как лежат на оси $z$ и не смещаются при изменении расстояния модуля измерения до поверхности. Тогда, учитывая поворот камеры $\alpha$ и ее смещение $d$ в форме матриц поворота

$$
R=\left|\begin{array}{lll}
r_{11} & r_{12} & r_{13} \\
r_{21} & r_{22} & r_{23} \\
r_{31} & r_{32} & r_{33}
\end{array}\right|
$$

и переноса

$$
T=\left|\begin{array}{l}
d \\
0 \\
0
\end{array}\right|
$$

соответственно, получим

$$
\begin{aligned}
& \left|\begin{array}{l}
x_{\mathrm{c}} \\
y_{\mathrm{c}} \\
z_{\mathrm{c}}
\end{array}\right|=R^{T} \cdot(P+T)=\left|\begin{array}{lll}
r_{11} & r_{12} & r_{13} \\
r_{21} & r_{22} & r_{23} \\
r_{31} & r_{32} & r_{33}
\end{array}\right| \cdot\left|\begin{array}{l}
d \\
0 \\
z
\end{array}\right|= \\
& =\left|\begin{array}{l}
r_{11} \cdot d+r_{13} \cdot z \\
r_{21} \cdot d+r_{23} \cdot z \\
r_{31} \cdot d+r_{33} \cdot z
\end{array}\right| .
\end{aligned}
$$

Подставляя (4) в (2), окончательно получим:

$$
\begin{aligned}
& u(z)=f \cdot \frac{r_{11} \cdot d+r_{13} \cdot z}{r_{31} \cdot d+r_{33} \cdot z}+c_{x} \\
& v(z)=f \cdot \frac{r_{21} \cdot d+r_{23} \cdot z}{r_{31} \cdot d+r_{33} \cdot z}+c_{y} .
\end{aligned}
$$

Из (5) видно, что выражения координат проекции луча в кадре имеют одинаковый знаменатель, а их числитель линейно зависит $z$. Из этого следует, что трек проекция лазера в кадре имеет прямолинейные вид. В силу этого положение на нем может быть представлено единственной координатой $w$ :

$$
w=\sqrt{\left(u-u_{0}\right)^{2}+\left(v-v_{0}\right)^{2}},
$$

где $u_{0}$ и $v_{0}$ координаты начала трека, описывающее одно из крайних положений. Таким образом, зависи-

мость проекции луча в кадре камеры от расстояния может быть представлена в виде функции

$$
z=f(w)
$$

Функцию (7) целесообразно определять путем аппроксимации экспериментальных данных в процессе калибровки системы. Следует отметить, что при этом могут быть также учтены и скомпенсированы нелинейные искажения оптической системы.

\section{3. Конструктивные параметры и особенности многолучевого модуля измерения}

В данной работе предполагается, что поверхность в локальной области измерения геометрически близка к плоскости. Для оценки её ориентации и положения необходимо измерить расстояние до неё в не менее, чем в трёх точках, следовательно, модуль измерения должен включать в себя не менее трех лазеров, одновременно наблюдаемых в кадре камеры. Для упрощения последующих вычислений лазеры размещаются параллельно.

Для корректной работы измерительного модуля проекции лазеров должны попадать в поле зрения камеры, причем для обеспечения наибольшей разрешающей способности их ход в кадре должен быть максимальным при изменении регистрируемого положения в рабочем диапазоне. В силу параллельности лучей лазеров последовательность их проекций в кадре камеры не меняется при изменении расстояния до поверхности, при этом их смещение происходит в одном направлении. Таким образом рабочий диапазон измерительного модуля, определяется положением крайних проекций лазеров по направлению движения. 


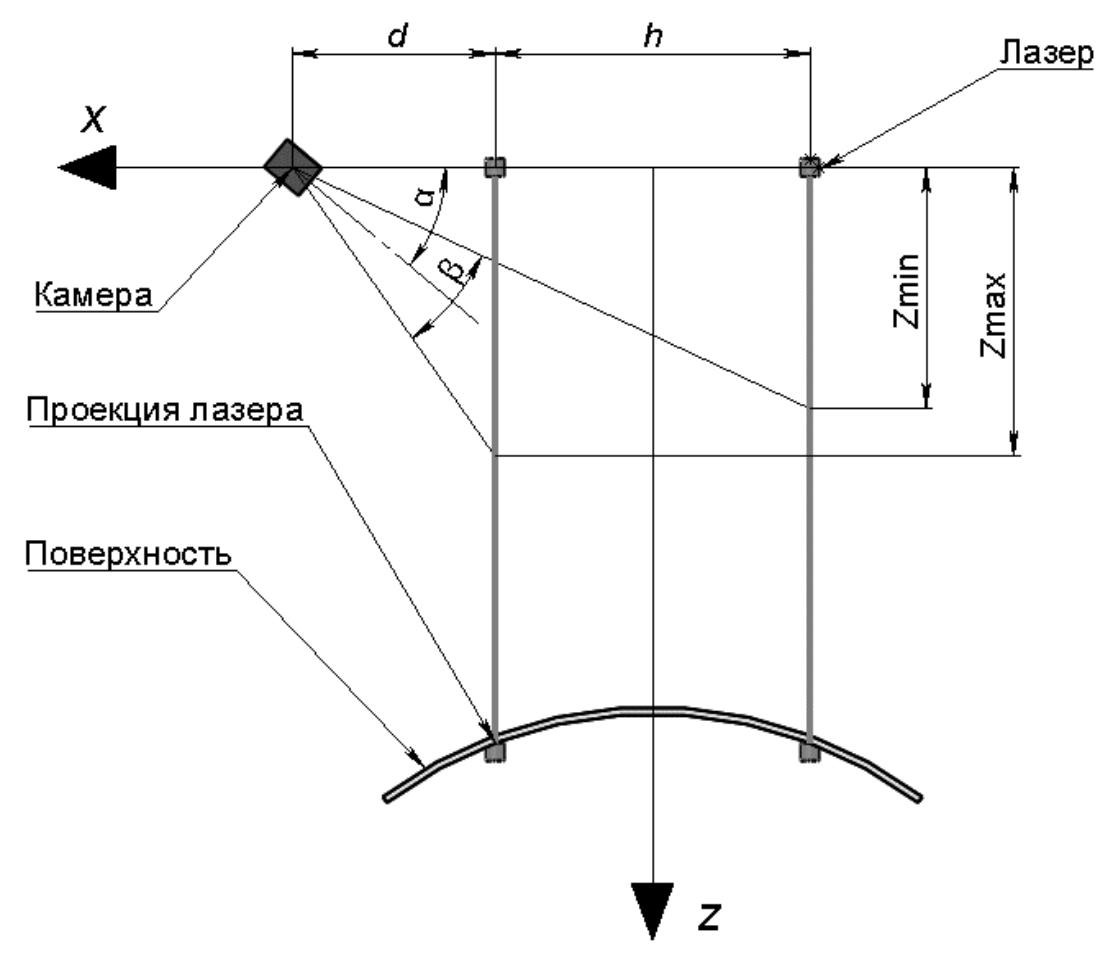

Рис. 2. Схема модуля измерения ориентации

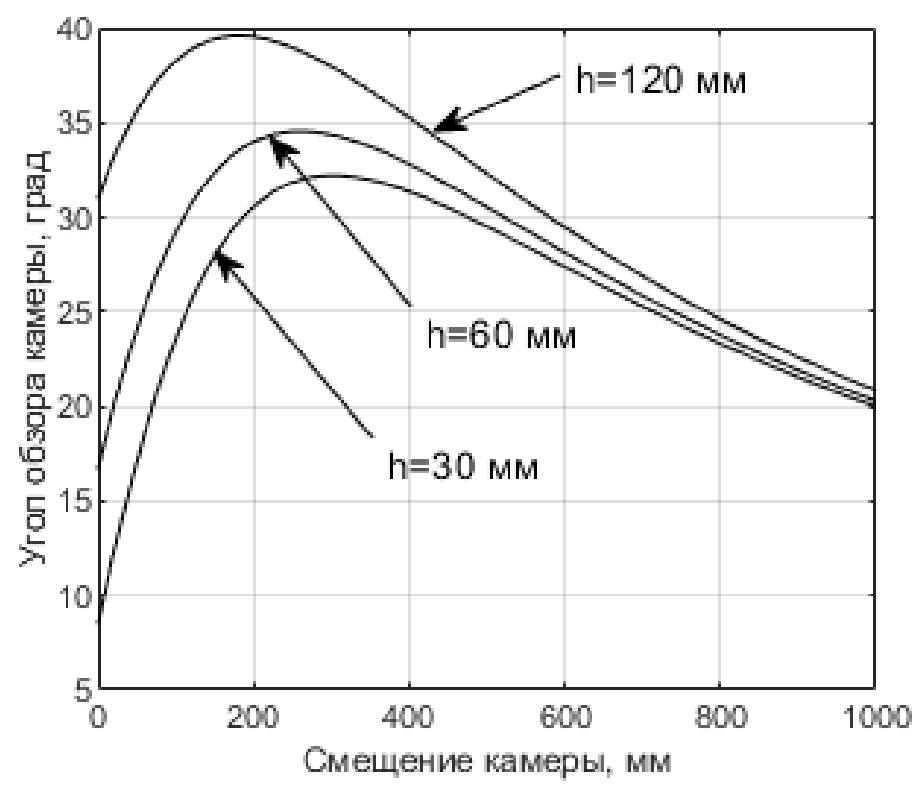

Рис. 3. Зависимость угла обзора $\beta$ от смещения камеры d

В соответствии с вышесказанным, рассматривается конструкция модуля измерения на рисунке 2 , где $h-$ расстояние между крайними лазерами.

В соответствии с конструкцией на рисунке 2 выражение (1) примет вид:

$$
\begin{aligned}
& (d+h) \cdot d+z_{\min } \cdot z_{\max }= \\
& =\sqrt{(d+h)^{2}+z_{\min }^{2}} \cdot \sqrt{d^{2}+z_{\max }^{2}} \cdot \cos \beta
\end{aligned}
$$

Выражение (8) связывает основные конструктивные параметры многолучевого измерительного модуля 


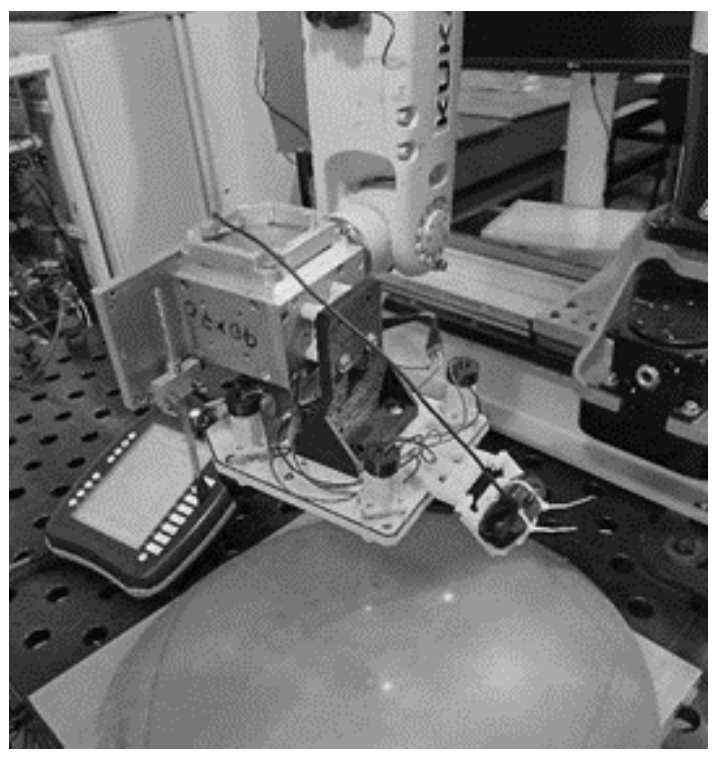

Рис. 4. Модуль измерения

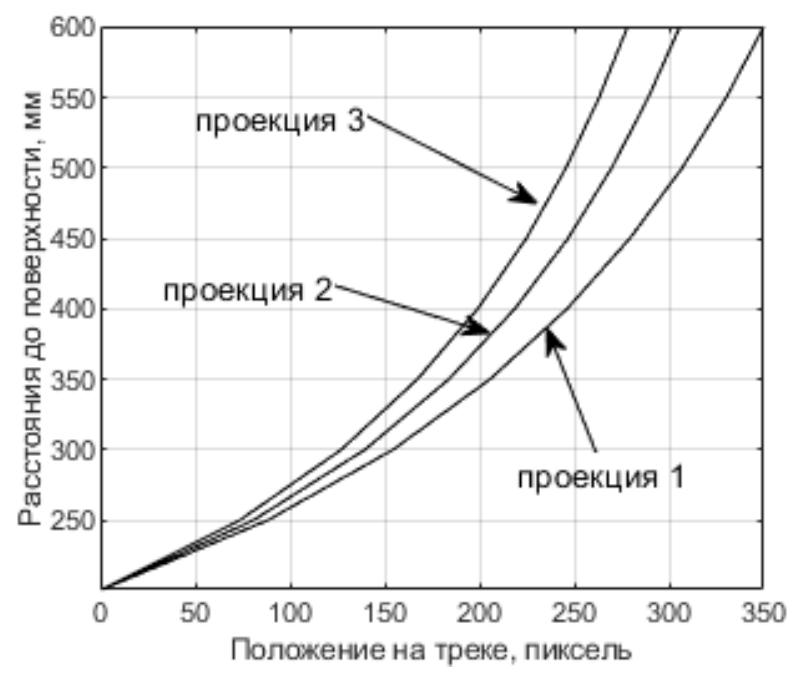

a)

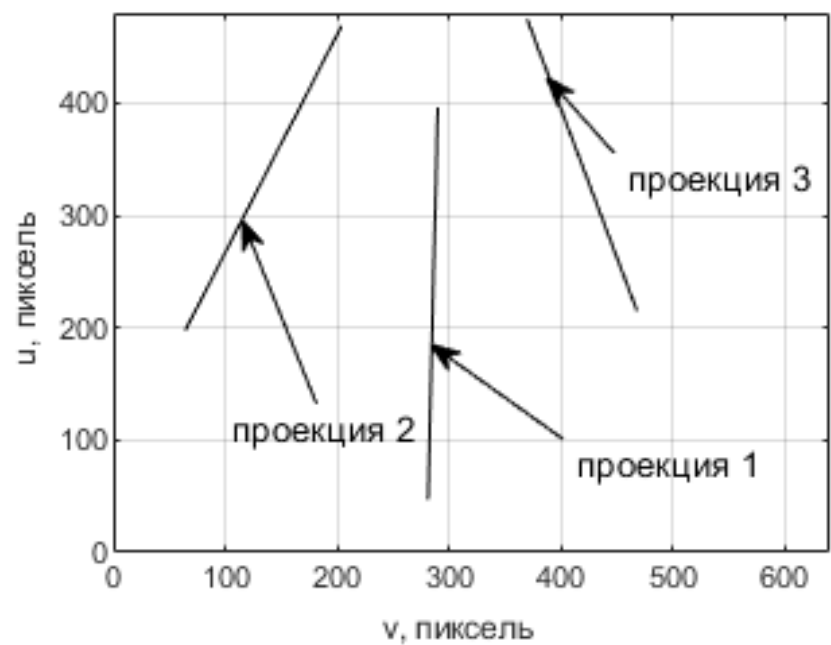

б)

Рис. 5. Результаты калибровка трехлучевого модуля измерения: а) калибровочная зависимость; б) треки проекций лазеров в кадре камеры

и позволяет вычислить оптимальное в соответствии с расчетной схемой значение любого из них, при условии, что остальные заданы. При этом угол наклона камеры $\alpha$ однозначно определяется из расчетной схемы в соответствии с выражением

$$
\alpha=\arctan \left(\frac{z_{\min }}{d+h}\right)+\frac{\beta}{2} .
$$

На рисунке 3 представлен пример зависимости угла обзора $\beta$ от смещения камеры $d$ при $z_{\min }=200$ мм, $z_{\text {max }}=600$ мм и различных $h$.
Представленные на графике зависимости отражают ситуацию, когда в кадре видно все проекции лазеров в рабочем диапазоне. Использование меньшего угла обзора $\beta$ не позволит попасть в кадр некоторым проекциям лазера, при этом больший угол обзора приведет к сокращению длины трека в кадре и снижению разрешающей способности. Также следует отметить, что в рассмотренном примере модуль измерения может быть реализован в виде двух конструктивных исполнений для угла обзора $\beta$, для которого существует два варианта смещения камеры $d$. Выбор конструктивного 


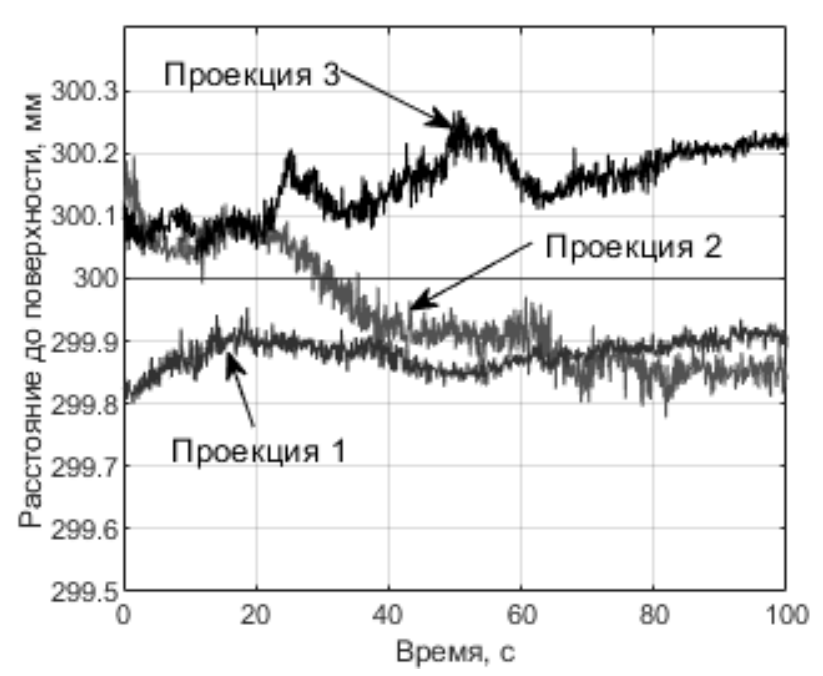

a)

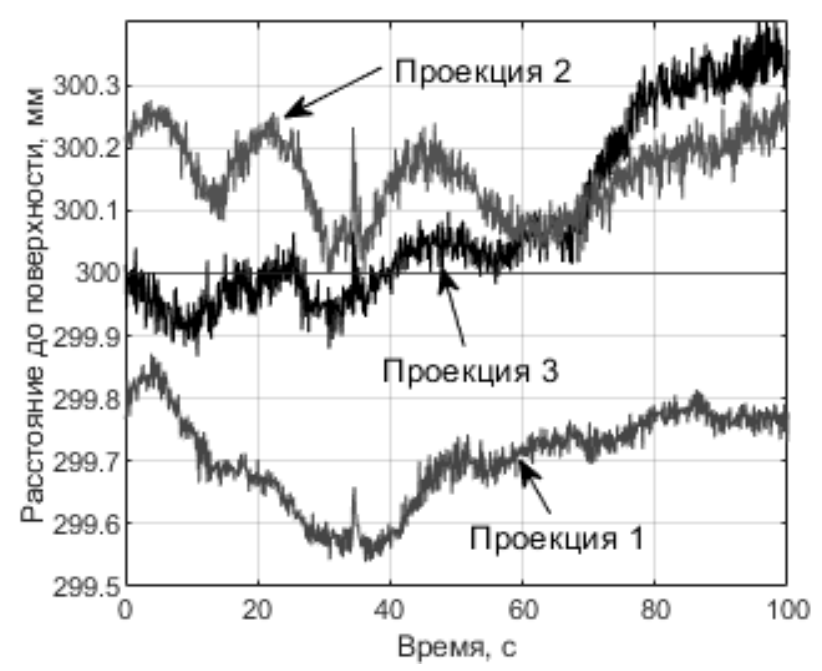

б)

Рис. 6. Результаты экспериментальных измерений: а) рабочий диапазон zmin = 200 мм, $z \max =600$ мм; б) рабочий диапазон zmin $=200$ мм, $\operatorname{zmax}=1000 \mathrm{Mm}$

исполнение может учитывать необходимость уменьшенных габаритов модуля измерения или лучшего визуального контроля. При изменении расстояния между лазерами $h$ картина качественно не меняется.

\section{4. Экспериментальное исследование}

Экспериментальный модуль измерения представлен на рисунке 4. Он реализован с использованием камеры с разрешением 640 на 480 пикселей и трёх красных лазеров. Измерительный модуль размещен на фланце робота KUKA KR10 R900.

Для калибровки модуля измерения его положение относительно перпендикулярной плоскости последовательно изменяется в рабочем диапазоне с помощью робота. При этом для каждого положения $z$ фиксируются координаты проекций лазеров $u, v$ в кадре камеры с помощью методов компьютерного зрения.

Индивидуальная для каждого лазера калибровочная зависимость получена путем аппроксимации экспериментальных данных степенным полиномом 6 порядка. Пример экспериментальных зависимостей для трех лазеров представлен на рисунке 5. Экспериментальные данные получены с шагом 50 мм в рабочем диапазоне $z_{\min }=200 \mathrm{mм}$, $z_{\text {max }}=600$ мм, при $\beta=30$ град, $h=60$ мм.

На разрешающую способность модуля измерения оказывает влияние рабочий диапазон, в соответствии с которым выбираются конструктивные параметры модуля. Для оценки этого влияния рассмотрим две конструкции измерительной системы с различными рабочими диапазонами. В первом случае он составляет $400 \mathrm{~mm}\left(z_{\min }=200 \mathrm{~mm}\right.$, $\left.z_{\text {max }}=600 \mathrm{Mм}\right)$, во втором $800 \mathrm{mм}\left(z_{\min }=200 \mathrm{mм}\right.$, $z_{\max }=1000$ мм). В рассматриваемых экспериментах рабочий диапазон обеспечен соответствующим положением камеры $d$ и углом ее наклона $\alpha$. После конфигурирования каждая система откалибрована.

На рисунке 6 представлены результаты сравнительного эксперимента, где рассматриваемые измерительные системы с помощью робота размещены и неподвижно зафиксированы на расстоянии 300 мм от перпендикулярной поверхности. В регистрируемых при этом показаниях дальномеров присутствуют выраженные осцилляции, в которых можно выделить высокочастотные и низкочастотные составляющие погрешности измерений.

Различия по разрешающей способности рассматриваемых измерительных систем преимущественно прослеживаются в амплитуде высокочастотной составляющей погрешности измерения. В системе с большей разрешающей способностью эта амплитуда оказывается меньше. Однако ее вклад в ошибку измерения оказывается не так существенен, как влияние низкочастотной составляющей погрешности. Эта составляющая преимущественно связана с нестабильностью источника лазерного излучения, а также с нестабильностью фоновой засветки изображения с камеры, которая параллельно используются для 
удаленного мониторинга области обработки. Эксперименты показали, что получаемая в таких условиях погрешность измерения составляет порядка $0.2 \%$ от рабочего диапазона.

При построении системы технического зрения, дополненная группой лазеров с параллельной ориентацией лучей, следует учитывать влияние таких факторов как качество камеры, стабильность работы лазерных источников, фоновой засветки и жесткости конструкции модуля.

\section{Зак^ючение}

В работе рассмотрены задачи позиционирования рабочего инструмента промышленного робота относительно наблюдаемого объекта и его окружения. Реализована и исследована система технического зрения, дополненная группой лазеров с параллельной ориентацией лучей. Определена зависимость координат проекции лазера в кадре камеры от расстояния до поверхности. Предложена техника калибровки измерительного модуля, учитывающая нелинейные искажения оптической системы. Предложен метод расчета конструктивных параметров системы, направленный на повышение её разрешающей способности. Проведено экспериментальное исследования модуля измерения, установленного на фланец промышленного робота KUKA KR10 R900. Эксперименты проведены для измерительных модулей с различными рабочими диапазонами. Выполнена оценка влияния конструктивных параметров модуля измерения на разрешающую способность. Достигнутая в рассматриваемом эксперименте точность измерения составила $0.2 \%$ от рабочего диапазона. Предложены рекомендации по построению измерительной системы.

\section{ЛИТЕРАТУРА}

1. Wenbin Zhang, Chengliang Zhang, Chengbin Li, He Zhang, "Object color recognition and sorting robot based on OpenCV and machine vision", IEEE, 2020 [2020 IEEE11th International Conference on Mechanical and Intelligent Manufacturing Technologies (ICMIMT)]

2. Baishali Chaudhury, "Single axis manipulator control using machine vision and virtual reality technique", IEEE, 2008 [2008 IEEE International Symposium on Industrial Electronics]

3. Barbara Araujo, Julierme Araujo, Rafael Silva, Danilo Ragis, "Machine Vision for Industrial Robotic Manipulator using Raspberry Pi", IEEE, 2018 [2018 13 th IEEE International Conference on Industry Applications (INDUSCON)]

4. M. Herman Jamaluddin, M. Azmi Said, Marizan Sulaimanm, Chong Shin Horng, "Vision Guided Manipulator for Optimal Dynamic Performance”, IEEE, 2006 [2006 4th Student Conference on Research and Development]

5. Priyanka Karuppiah, Hem Metalia, Kiran George, "Automation of a wheelchair mounted robotic arm using computer vision interface", IEEE, 2018 [2018 IEEE International Instrumentation and Measurement Technology Conference (I2MTC)]

6. Rodrigo Melo, Thelmo Ponted de Araujo, Arata Andrade Saraiva, Jose Vigno Moura Sousa, Nuno Miguel Fonseca Ferreira, "Computer Vision System with Deep Learning for Robotic Arm Control", IEEE, 2018 [2018 Latin American Robotic Symposium, 2018 Brazilian Symposium on Robotics (SBR) and 2018 Workshop on Robotics in Education (WRE)]

7. Emre Dandil, Kerim Kursat Cevik, "Computer Vision Based Distance Measurement System using Stereo Camera View", IEEE, 2019 [2019 3rd International Symposium on Multidisciplinary Studies and Innovative Technologies (ISMSIT)]

8. K. Karthika, S. Adarsh, K.I. Ramachandran, "Distance Estimation of Preceding Vehicle Based on Mono Vision Camera and Artificial Neural Networks", IEEE, 2020 [2020 11th International Conference on Computing, Communication and Networking Technologies (ICCCNT)]

9. Viacheslav V. Kapustin, Yuri I. Maltsev, Andrey K. Movchan, "Controlling a Robotic Manipulator Using Optical Feedback”, IEEE, 2019 [2019 International Siberian Conference on Control and Communications (SIBCON)]

10. Dongdong Chen, Peijiang Yuan, Tianmiao Wang, Ying Cai, Haiyang Tang, "A Normal Sensor Calibration Method Based on an Extended Kalman Filter for Robotic Drilling", MDPI Sensors, 2018.

11. Yuhao Gao, Dan Wu, Chenggen Nan, Ken Chen, "Normal direction measurement in robotic drilling and precision calculation", The International Journal of Advanced Manufacturing Technology, pp. 1311-1318, 2015

12. Yuhao Gao, Dan Wu, Yunfei Dong, Xinguo Ma, Ken Chen, "The method of aiming towards the normal direction for robotic drilling", The International Journal of Advanced Manufacturing Technology, pp. 787-794, 2017

13. Tian Wei, Zhou Weixue, Zhou Wei, Liao Wenhe, Zeng Yuanfan, "Auto-normalization algorithm for robotic precision drilling system in aircraft component assembly", Chinese Journal of Aeronautics, pp. 495-500, 2013

14. Yun Bo Bi, Yong Chao Li, Yi Hang Jiang, Jiang Xiong Li, Wei Wang, Mian Gao, Shuo Li, "An Industrial Robot Based Drilling System for Aircraft Structures”, Applied Mechanics and Materials, pp. 151-157, 2013

15. ZhaoCai Du, Yanbin Yao, "Measurement Method for Evaluating Normal Direction of Surface for Digital Drilling and Riveting", SAE Technical Papers, 2012 [SAE2012 Aerospace Manufacturing and Automated Fastening Conference \& Exhibition]

16. Peijiang Yuan, Qishen Wang, Tianmiao Wang, Chengkun Wang, Bo Song, "Surface normal measurement in the end effector of a drilling robot for aviation", IEEE, 2014 [2014 IEEE International Conference on Robotics and Automation (ICRA)] 
17. Dongdong Chen, Peijiang Yuan, Tianmiao Wang, Zhenyun Shi, Yuanwei Liu, Minqing Lin, "Perpendicularity adjustment end effector for aeronautical drilling robot", IEEE, 2016 [2016 IEEE/RSJ International Conference on Intelligent Robots and Systems (IROS)]

18. Dongdong Chen, Peijiang Yuan, Tiammiao Wang, Qishen Wang, Chengkun Wang, Fengchao Wang, "Intelligent surface normal measurement method of end effector for the aeronautical drilling robot", IEEE, 2014 [2014 International Conference on Multisensor Fusion and Information Integration for Intelligent Systems $(\mathrm{MFI})]$

19. Maozhen Gong, Peijiang Yuan, Tianmiao Wang, Leibin Yu, Hongwen Xing, Wen Huang, "A novel method of surface-normal measurement in robotic drilling for aircraft fuselage using three laser range sensors", IEEE, 2012 [2012 IEEE/ASME International Conference on Advanced Intelligent Mechatronics (AIM)]

20. Peiliang Yuan, QiShen Wang, ZhenYun Shi, TianMiao Wang, ChengKun Wang, DongDong Chen, LiHeng Shen, "A micro-adjusting attitude mechanism for autonomous drilling robot end-effector", Science China Information Sciences, pp. 1-12, 2014

21. Rui Zhang, PeiJiang Yuan, Maozhen Gong, "Intelligent Surface-normal Adjustment System and Application in Aeronautical Drilling Robot", IEEE, 2012 [2012 Second International Conference on Intelligent System Design and Engineering Application]

22. J. Matteo Munaro, Edmond Wai Yan So, Stefano Tonello, Emanuele Menegatti, "Efficient Completeness Inspection Using Real-Time 3D Color Reconstruction with a Dual-Laser Triangulation System" on Integrated Imaging and Vision Techniques for Industrial Inspection, 2015, pp. 201-225.

23. Peter Sturm, "Pinhole Camera Mode" on Computer Vision, 2014

( Ф Филонов Иван Владимирович ( Filonov.iv@dvfu.ru),

Ноткин Борис Сергеевич ( notkin@iacp.dvo.ru ), Змеу Константин Витальевич ( zmeu.kv@dvfu.ru).

Журнал «Современная наука: актуальные проблемы теории и практики»

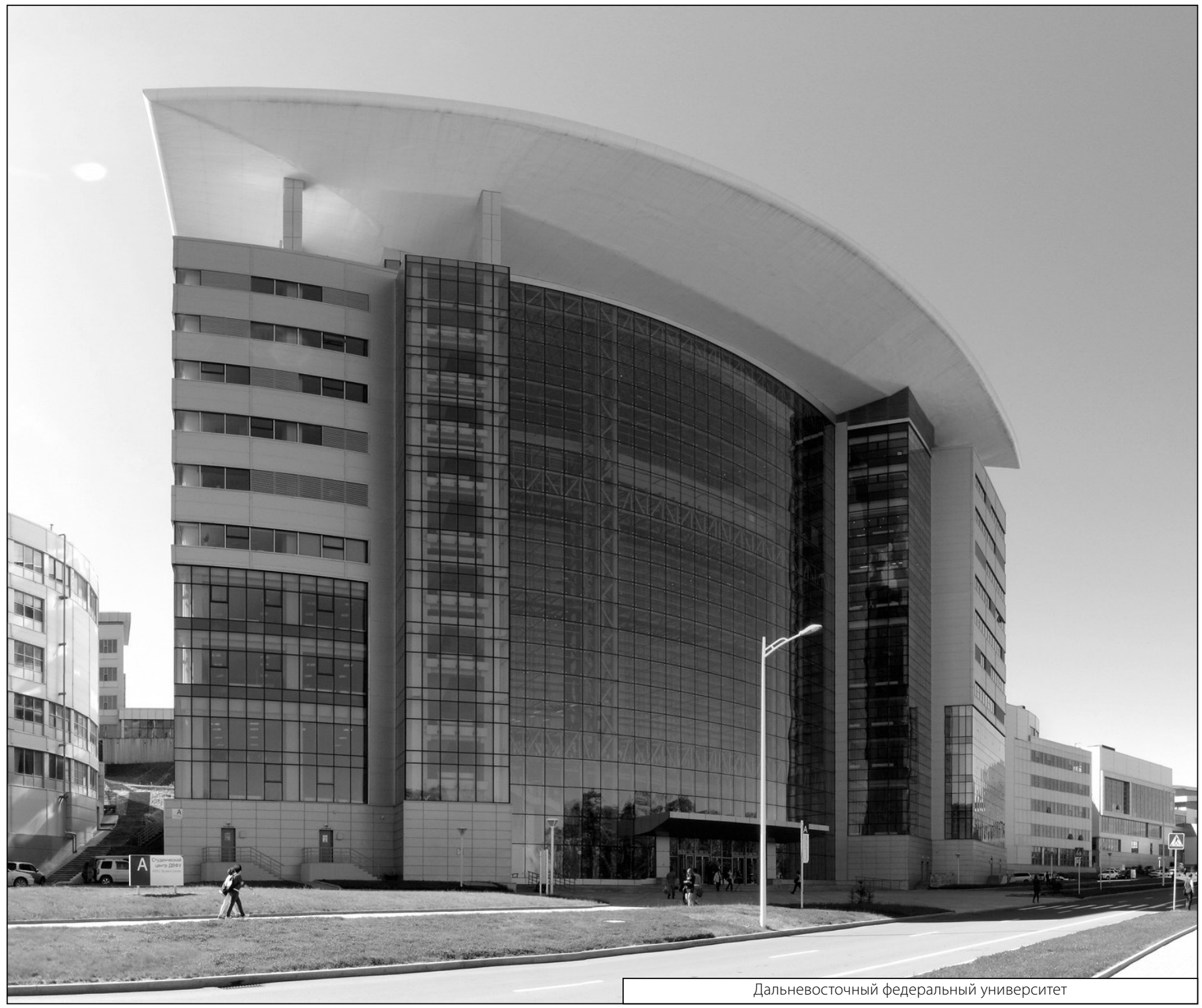

